\title{
The Source of a coronal mass ejection in a decayed solar active region
}

\author{
Debi Prasad Choudhary, Nandita Srivastava, and Sanjay Gosain
}

Udaipur Solar Observatory, Physical Research Laboratory, Post Box No. 198, Udaipur, 313 001, India

Received 1 July 2002 / Accepted 21 August 2002

\begin{abstract}
We have studied the source of a coronal mass ejection (CME), which occurred in a decayed active region NOAA 7978 on 19 October, 1996. The active region NOAA 7978 first appeared on the solar disk on 2 June, 1996 and made more than five disk passages before it decayed. The event analysed in this paper was observed during fifth disk passage. We have attempted to identify the mechanism responsible for triggering this CME based on the analysis of photospheric magnetograms (MDI/SoHO), chromospheric filtergrams (Meudon and Big Bear Observatories) and coronal images (SXT/Yohkoh). We found that the emergence of new bipoles in the active region led to the eruption of a low-lying sheared filament observed in SXT images, subsequently followed by filament eruption observed both in $\mathrm{H}_{\alpha}$ and EUV wavelengths (EIT/SoHO). The study aims at chronologically investigating the occurrence of the events in different wavelengths, in order to have a comprehensive understanding of the mechanism involved in the launch of the CME.
\end{abstract}

Key words. Sun: coronal mass ejection - Sun: activity - Sun: X-rays - Sun: magnetic field

\section{Introduction}

Coronal mass ejections (CMEs) are the most dynamic and energetic aspects of solar activity, which affect the terrestrial environment. CMEs are closely related to a variety of phenomena such as Long Duration Events (LDEs), sprays, erupting loops and filaments (Svestka 2001; Sheeley et al. 1983). It is also observed that CMEs without flares are qualitatively similar to CMEs with flares, except that they are accelerated to high speed at much lower heights (MacQueen \& Fisher 1983; Kahler 1992). Therefore, identifying a mechanism for the trigger of any CME may have wide implications. The CMEs are believed to occur mainly as the stressed magnetic structure becomes unstable and eventually explode. The instability in the magnetic structures can be initiated due to the density fluctuation in prominence material, slowly-moving the magnetic foot-points at the photosphere (Barnes \& Sturrock 1972) or by injecting the poloidal magnetic flux into the active region (AR) magnetic structure (Chen 2001). As a result of the instability, magnetic explosion of "core field" near the neutral line of an active region may disrupt the "envelope field" leading to a CME (Moore et al. 2001).

The X-ray observations obtained from Yohkoh have been used to understand the behavior of the low corona during the launch of a CME, specially for identifying the on-disk CMEs. Consequently, there have been many studies to identify the source of CMEs in X-ray emission. Hudson (1998) has reviewed the impact of observations of CME at high

Send offprint requests to: Debi Prasad Choudhary, e-mail: debi@prl.ernet.in temperature. The careful analysis of the X-ray image morphology of an LDE observed by Yohkoh showed that although the basic ingredients of the standard model are consistent with the observations, there exists conflicts. For example, as the CME launch takes place far away from the flare core, the relation of the associated reconnected field lines with the flare are not clear. Further, the flare occurs well after the CME launch and the associated reconnection jets or slow shock fronts are not clearly observed (Hudson et al. 1996). The observations also show that the source of many CMEs appear as long-lived sigmoid structures in the soft X-ray images of solar corona (Sterling \& Hudson 1997). The other soft X-ray signatures of the CMEs are "dimming" and "transient coronal hole" at the source region (Hudson 1998; Smith et al. 1997). It is also observed that the shock waves generated by the flares destabilize the large coronal structures, such as trans-equatorial interconnecting loops, leading to CMEs (Khan \& Hudson 2000). However, several questions related to the source of CME still remain unanswered. For example, the cause of explosion of the "core field" is not clear. Similarly, the plasma conditions of the active region at the time of the beginning of CME are obscure.

In this paper, we analysed the Yohkoh SXT observations related to a CME of 19 October 1996 observed by LASCO coronagraphs on board the Solar and Heliospheric Observatory (SoHO) spacecraft. The investigation is based on several data sets obtained in different wavelengths, which yield specific information on the CME mechanism. The Michelson Doppler Imager (MDI/SoHO) images have been used to infer the flux emergence in the photosphere. The filament eruption observed in $\mathrm{H}_{\alpha}$ filtergrams recorded by Meudon and Big Bear Solar 
Observatories have also been used to study the associated chromospheric activity related to the CME. Our study based on these data sets leads to the inference of plasma temperature of the active region and the possible mechanism for the sheared core field explosion which might be the prime cause for CME initiation.

\section{The active region NOAA 7978 and the CME of 19 October 1996}

The AR NOAA 7978 was one of the best observed solitary active regions, as it appeared in the minimum phase of the solar cycle 22 (Hudson et al. 1998). It survived over more than five solar rotations and was named as NOAA 7981 and 7986 in successive rotations. The sunspots in this active region first appeared on 6 July 1996 and attained an area of 400 millionth of solar disk area within four days. During the subsequent solar rotations the $\mathrm{AR}$ evolved from complex ( $\beta \gamma \delta$ class) to simplified magnetic structure generating an X-class flare, 52 other types of flares and Long Duration Events (LDEs) and 26 CMEs. The energy release in this process could be due to helicity shedding. As the total helicity shed by the active region was more than what can be injected by differential rotation alone, the main source could be the inherent twist of the magnetic flux tubes forming the active region (Démoulin et al. 2002). This being the only active region on the solar disk during July-October 1996, the study of the CMEs, associated with it, can be made to isolate the exact cause of the trigger. By the fifth apparition of the AR in October 1996, the spots had decayed and the three-dimensional magnetic field configuration had relaxed to a potential configuration (Choudhary et al. 2001).

A large CME was observed on 19 October 1996 by all the three LASCO coronagraphs on SoHO, when the active region had decayed considerably, followed by a filament eruption (Plunkett et al. 1997). The gradual phase of the event started associated with the AR with a slow rise of a pre-existing loop recorded in LASCO-C1 on 18 October, 1996 and was seen up to a height of $1.8 R_{\odot}$. On 19 October, the expansion and eruption of this loop took place between 15:05-21:19 UT, during which the $\mathrm{H}_{\alpha}$ filament became activated and erupted. The white light CME (LASCO-C2 and C3) data showed that the leading edge traveled outwards at a speed of about $490 \mathrm{~km} \mathrm{~s}^{-1}$ to a distance of $30 R_{\odot}$. However, in the inner corona (i.e. in LASCOC1) the speed was estimated as $25 \mathrm{~km} \mathrm{~s}^{-1}$, which means the CME material was accelerated at approximately $6 \mathrm{~m} \mathrm{~s}^{-2}$ in the lower corona.

\section{Observational data}

The Yohkoh data obtained by the soft X-ray telescope (SXT) in full disk half and quarter resolution and partial field mode were used to study the pre-CME X-ray signature of the event. The data analysis was accomplished using the IDL-based Solarsoft package. The individual frames were corrected for flat-field and saturated pixels, following which registration and normalization was carried out taking into account the spacecraft vibrations. The longitudinal photospheric magnetograms were obtained from the observations made by MDI/SoHO. The detailed analysis of the CME observed by LASCO C1, C2 and C3 coronagraphs has been made by Plunkett et al. (1997). Therefore, we have not analysed the CME data in this paper. We have used the full-disk $\mathrm{H}_{\alpha}$ filtergrams from Paris-Meudon and Big Bear observatories to determine the time of filament eruption.

\section{Discussion}

\subsection{GOES $X$-ray time profile and $H_{\alpha}$ filament eruption}

The full disk integrated $\mathrm{X}$-ray flux as a function of time during 19-20 October 1996, observed in 1-8 $\AA$ and 0.5-4 $\AA$ GOES channels, is shown in Fig. 1. As NOAA 7978 was the only active region on the solar disk during the time of observation, any fluctuation in the X-ray intensity time profile can be attributed to the activities occurring at this site. Accordingly, we find that a B6.0 class long duration event (LDE) was associated with the CME, which started at 16:30 UT on 19 October 1996 and lasted for more than 10 hours. The $0.5-4 \AA$ GOES channel registered the flux only during the pre-flare phase. In this wavelength range $\mathrm{Ca}$ XIX and Fe XXV are the main sources of $\mathrm{X}$-ray flux. As, Ca XIX is excited at a lower temperature than that required for exciting Fe XXV, the observed GOES time profiles imply that the plasma temperature of the flare was at least $16 \times 10^{6} \mathrm{~K}$ in the initial phase (Simnett 1991; Antonucci et al. 1984). During the rest of the time the flare emitted only thermal X-ray radiation. Although no flare was recorded in $\mathrm{H}_{\alpha}$, towards the end of the initial hot pre-flare phase a large $\mathrm{H}_{\alpha}$ filament situated in the dispersed flux of the active region was observed to erupt as shown in Fig. 2. A brightening in $\mathrm{H}_{\alpha}$ was observed near the location 2, where bipolar flux emerged associated with compact X-ray brightening. The initial activation of the filament took place between the regions marked 1 and 3 in Figs. $2 \mathrm{a}$ and $\mathrm{b}$. This is the only $\mathrm{H}_{\alpha}$ signature of the CME. As the filament material is also observed in SoHO-EIT (195 ̊) images, it indicates that the plasma is partially heated to a least a temperature of about $1.5 \times 10^{6} \mathrm{~K}$.

\subsection{Photospheric magnetograms}

It has been reported that there is a change in photospheric magnetic flux during the CME (Lara et al. 2000). We examine MDI magnetograms obtained around the time of CME to find the related photospheric magnetic activity. In early July 1996, the AR NOAA 7978 appeared as a compact active region and later showed a distinct evolutionary trend during the five successive solar rotations (Choudhary et al. 2001). As the active region decayed, the total X-ray flux reduced and the magnetic field of opposite polarity dispersed. The longitudinal magnetograms observed with the SoHO-MDI around the time of the flare are shown in Fig. 3. Compact bipoles, as shown by arrows in Fig. 3, appeared near the neutral line during the preflare phase of the LDE on 19 October 1996. These bipoles were found absent in the magnetograms obtained after the CME i.e., on 20 October 1996. The emergence of bipoles near the neutral line could be one of the mechanisms to destabilize the filament foot-points leading to its eruption. The change in the 


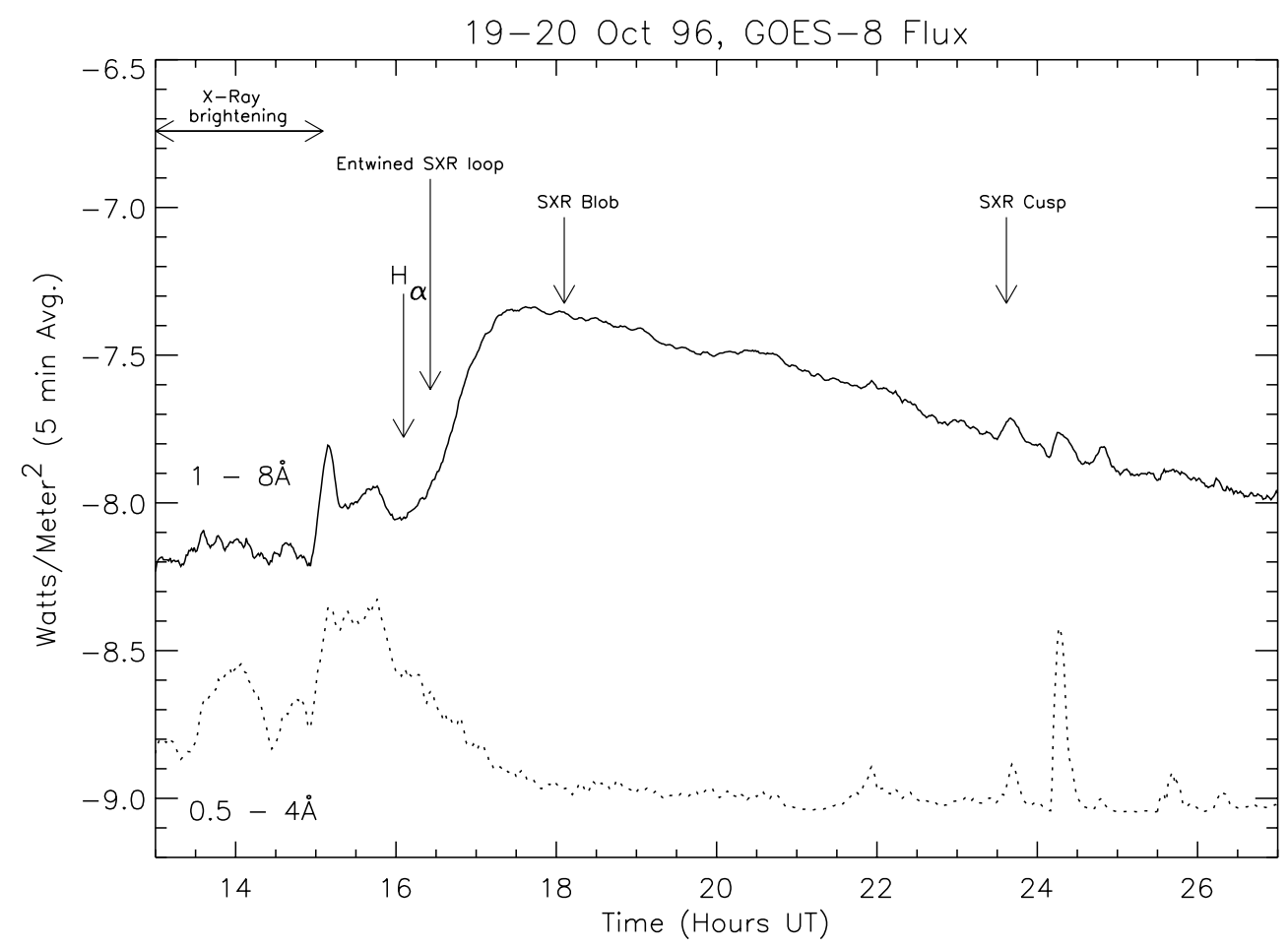

Fig. 1. X-ray flux as recorded by GOES-8 plotted against time. The solid and dotted lines are the flux recorded in $1-8 \AA$ and $0.5-4 \AA$ channels. Arrows indicate the timings of occurrence of various phenomena in $\mathrm{H}_{\alpha}$ and Yohkoh SXT images. The LDE associated with the CME of 19 October 1996 occurred at 16:30 UT. The pre-flare phase of LDE was observed during 14-16 UT in which the flux in channel 0.5-4 A is prominently seen.

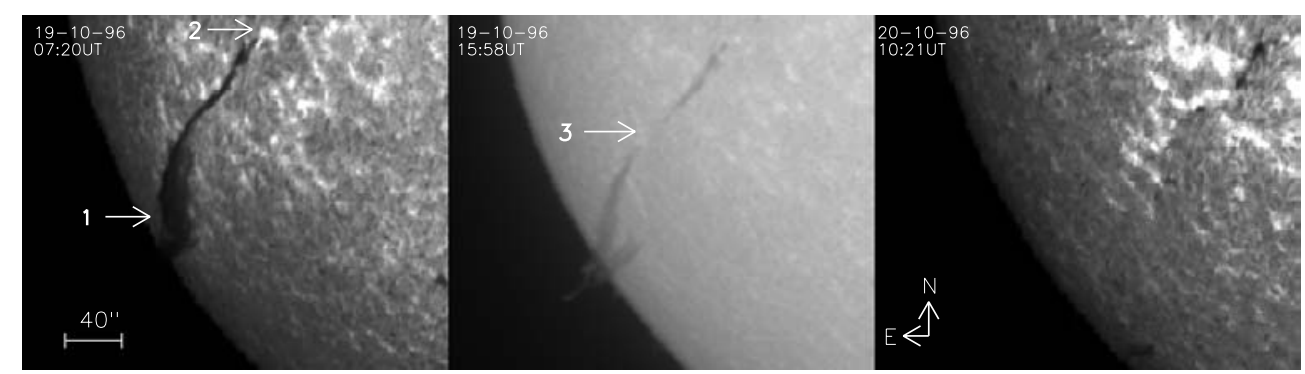

Fig. 2. (a), b), c) from left to right) Time lapse images recorded in $\mathrm{H}_{\alpha}$ show the eruption of a large stable filament on the SE limb. The left and right frames are from Meudon and the middle frame was recorded at Big Bear Solar Observatory.

photospheric magnetic flux associated with the CME could therefore be due to the emergence and decay of the bipoles.

\subsection{Yohkoh X-ray observations}

The Yohkoh SXT images provide vital clue about the energetic events leading to CME. Coinciding with the locations of the small bipoles, the co-temporal X-ray images display compact bright blob like structures as shown in Fig. 4. The blobs in the Fig. 4a, marked 1 and 2 were seen to be associated with the straight jets in the south-east direction, indicating high-speed plasma. The blob marked 2 coincides with the bipole identified with an arrow mark in the first frame of the Fig. 3. This blob was long lasting which can be traced in the 13:52:59 UT frame and later in 16:29:31 frame (marked 2 in Figs. 4 and 5). Harvey (1997) established a one-to-one association in the quiet sun. But, the role of the flux emergence in destabilizing the magnetic structures when they emerged near the active region neutral line is not clear. In Fig. 4b, we noticed bright parts of loop like structures associated with the blobs as marked 3 and 4 . The sequence of events in soft X-ray, leading to the filament eruption and CME are given in Fig. 5. In Fig. 5a, a group of bright loops were observed. Some of the foot-points of the loop are connected to the X-ray blobs described earlier. The loop structure appeared as a "8-like feature". The lower part of which was approximately $8.5 \times 10^{4} \mathrm{~km}$ (identified as 1) and was brightened in the initial phase. The total length of the loop is about $2.5 \times 10^{5} \mathrm{~km}$. Figure $5 \mathrm{~b}$ shows that after about one and half-hour, a bright blob like structure of $6.8 \times 10^{4} \mathrm{~km}$ in size (identified as 3) developed near the loop top and eventually erupted. In the later phase the well-known cusp structure of $1.7 \times 10^{5} \mathrm{~km}$ projected length was formed near the eruption region (Fig. 5c).

The chronology of events observed by Yohkoh SXT may be used to identify the physical processes leading to the observed CME. As shown in Fig. 1, SXR blob was noticed 


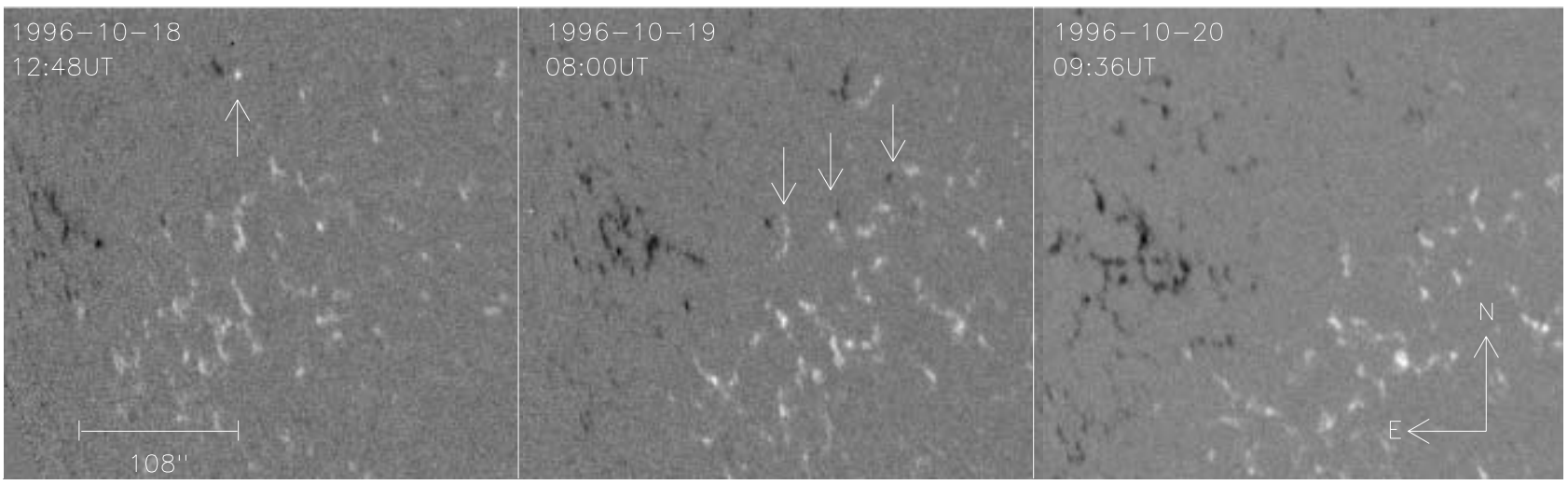

Fig. 3. (a), b), c) from left to right) The SoHO MDI longitudinal magnetograms of the active region NOAA 7978.

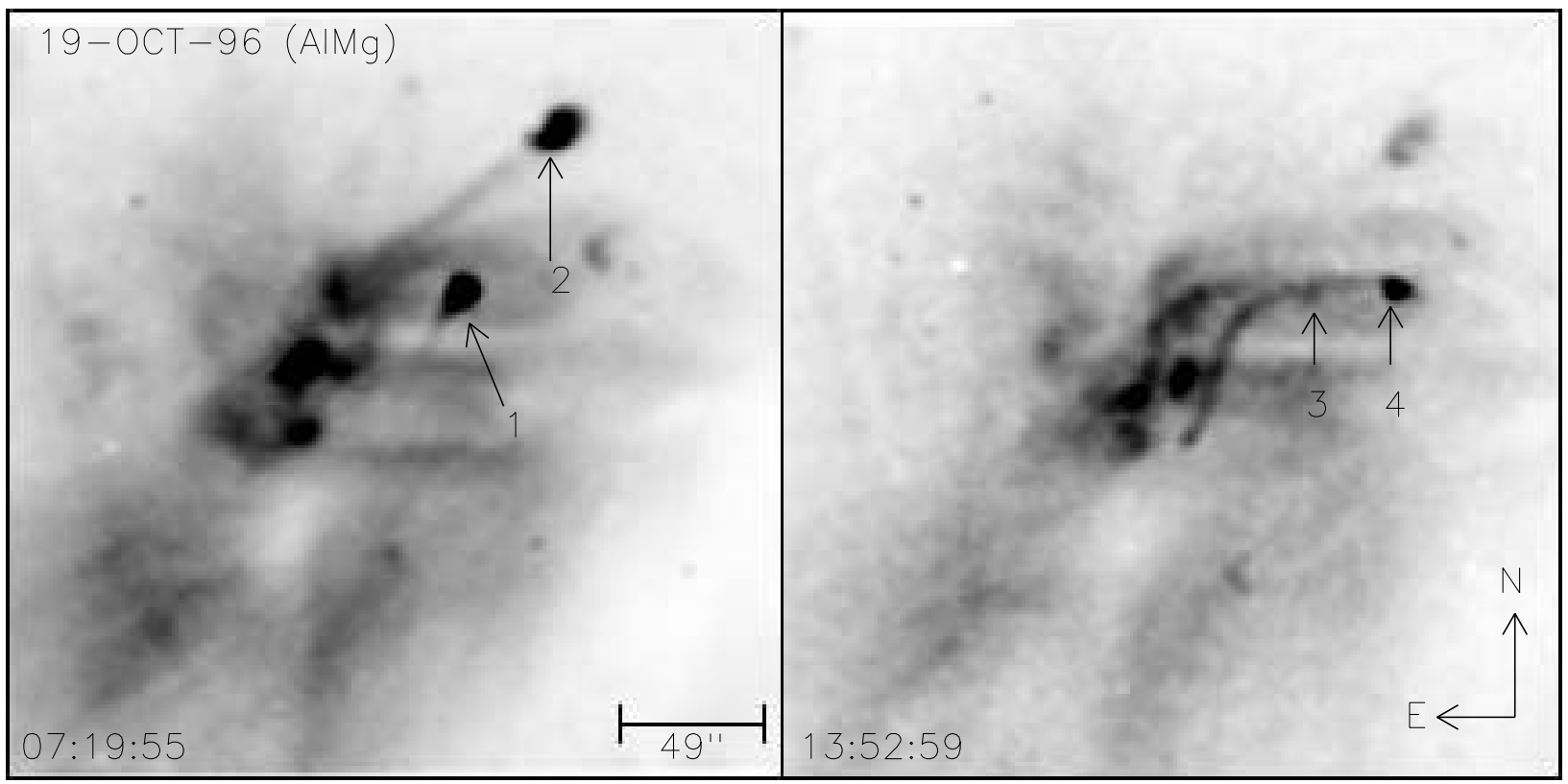

Fig. 4. (a), b) from left to right) Yohkoh SXT images of the active region NOAA 7978 prior to the CME.

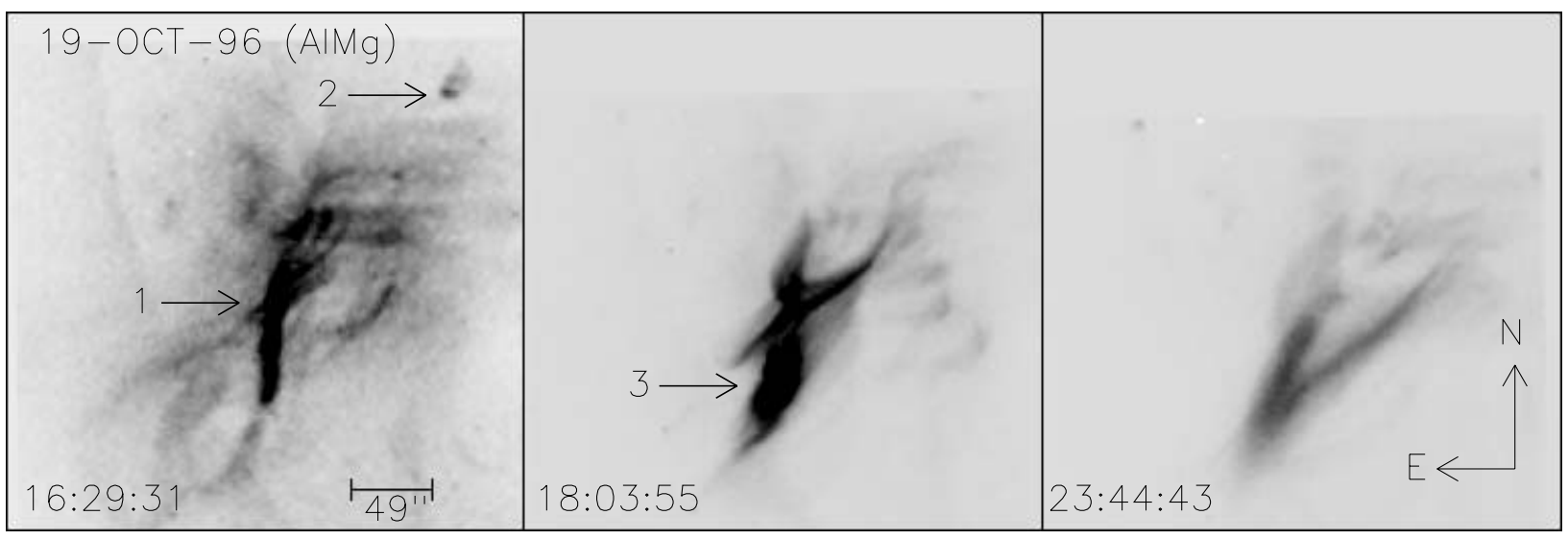

Fig. 5. (a), b), c) from left to right) Yohkoh SXT images of the active region NOAA 7978 during the CME.

following the localized X-ray brightening, $\mathrm{H}_{\alpha}$ filament eruption and appearance of entwined SXR loop respectively. The localized X-ray brightening were cotemporal with the disappearance of emerging small magnetic bipoles. This sequence of events may be understood with the following scenario.
The magnetic bipole annihilation release energy at lower solar atmosphere and increase the local gas pressure. Consequently, the heated plasma may be injected into the magnetic field structure. The observed blob could be the ejection of the hot material after gaining sufficient energy from the active region magnetic 
field structure. During this process, the magnetic field structure gets destabilized leading to filament eruption.

\section{Results and conclusions}

The 19 October 1996 CME was associated with a weak LDE. The basic mechanism governing the LDEs and compact impulsive flares is the same, namely the reconnection at chromospheric heights. The only difference between the two is that in the case of LDEs, the dissipation region is smaller (Blackman 1997). Further, in case of LDEs the heating might take place by reconnection well above the chromospheric heights. Recently, many theoretical models have been proposed which explain only a part of the observed features of CMEs. The theoretical and numerical models on CME can be divided into two groups. One group of models advocate for highly twisted structures called flux ropes (Gibson \& Low 1998; Amari et al. 2000). The other group argues for the plasma configurations consisting of low-lying sheared magnetic arcades (Antiochos et al. 1999). The twisted flux ropes are generated by shearing photospheric motion and generate the S-like structures resembling the sigmoid as seen in SXT images (Amari et al. 2000). In this model, the explosion of the core field is the cause of the CME (Moore et al. 2001). At some critical twist these structures erupt giving rise to CMEs. Similar motions can also produce low-lying sheared and high potential field lines (Tokman \& Bellan 2002).

Another important feature of CME is the association of prominence eruption (Gilbert et al. 2000). The prominence may erupt due to loss of equilibrium when magnetic energy exceeds a critical value following the imbalance between magnetic tension and compression. By reconnecting the fields in the photosphere one can increase the current in a pre-existing coronal current filament until the current becomes so strong that the balance between magnetic compression and tension force acting on the filament is suddenly lost (Forbes \& Isenberg 1991).

In the present case, we noticed emerging photospheric flux, sheared SXT loop structure and filament eruption. We did not notice any organised photospheric motion in and around the active region, which is required for the flux rope models. Alternatively, the explosion of low lying sheared magnetic arcades have been observed to be accompanying the CMEs (Moore et al. 2001). The associated LDEs are observed to get triggered by explosion of low-lying sheared filaments (Choudhary \& Gary 1999).

Based on the discussions given in the previous paragraphs, we describe a possible scenario taking into account the sequence of events given in Table 1. In the beginning the flux emerges at distinct points parallel to the neutral line. Followed by the flux emergence, the field symmetry breaks as opposite polarity migrate towards their respective pre-existing regions (Manchester 2001). A part of the emerged flux may also annihilate to heat the photospheric and lower chromospheric material. Consequently, the filament gets destabilized as the supporting magnetic structure is disturbed. This would lead to the instability of the magnetic field structure supporting the filament material and its eruption (Kahler 1992; Plunkett et al. 1997). Our observations also show that the emergence of the flux takes place over a time-scale of 6-7 hours, which is consistent with the
Table 1. Sequence of events during the CME of 19 October 1996.

\begin{tabular}{ll}
\hline \hline Time (UT) & Sequence of events \\
\hline 4:00 & Photospheric flux emergence \\
11:34-15:00 & SXR loop brightening \\
15:00 & Pre - flare spike observed by GOES \\
15:00-17:00 & EIT filament disruption \\
16:10 & $H_{\alpha}$ filament activation \\
16:27 & "8" like structure formation $(S X T)$ \\
16:25 & Beginning of B0.6 class LDE* \\
17:07 & CME onset ${ }^{* *}$ \\
17:20 & Peak of LDE \\
18:03 & Loop top blob formation \\
23:44 & Cusp formation \\
\hline
\end{tabular}

* As Published in Solar Geophysical Data, 1996, 627, 1.

** Derived by Plunkett et al. (1997).

earlier observations (Subramanian \& Dere 2001). At this time, following the eruption of $\mathrm{H}_{\alpha}$ filament, we noticed the entwined soft X-ray loop structure and the CME in the LASCO coronagraph. The X-ray blob structure developed only about one and half hour after the initiation of CME. Therefore, the blob is not a part of CME trigger but results due to overall destabilization of active region magnetic structure.

Our analysis of SXT images, photospheric magnetograms and $\mathrm{H}_{\alpha}$ filtergrams at the time of 19 October 1996 B6.0 flare gave a unique opportunity to trace the CME triggering mechanism. The summary of our analysis is given below.

1. The emergence of bipoles along the magnetic neutral line of the decayed active region NOAA 7978 destabilized its magnetic configuration. Some of these bipoles emerged near the foot-points of the sheared low-lying loops.

2. The destabilizing process brightened the sheared low-lying loops and led to the ejection of filament.

3. Coinciding with the filament eruption the entire magnetic configuration was destabilized, initiating the CME.

4. The blob like structure in SXT image was ejected much after the start of CME.

5. These observations support the magnetic explosion of the core sheared field in the active regions as the main cause for triggering the CME.

Acknowledgements. One of the authors (DPC) was on sabbatical leave from Physical Research Laboratory, Ahmedabad, India during the period of this work. He acknowledges the financial assistance provided by the National Astronomical Observatory of Japan during his stay at Mitaka. He also acknowledges the help extended by Professor T. Kosugi and Dr. Josef I. Khan at ISAS, Sagamihara during the Yohkoh data reduction. We thank the referee for the comments, which were helpful to improve some part of the paper.

\section{References}

Amari, T., Luciani, J. F., Mikic, Z., \& Linker, J. 2000, ApJ, 529, L49 Antiochos, S. K., DeVore, C. R., \& Klimchuk, J. A. 1999, ApJ, 529, L49

Antonucci, E., Gabriel, A. H., \& Dennis, B. R. 1984 ApJ, 510, 485

Barnes, C. W., \& Sturrock, P. A. 1972, ApJ, 174, 659

Blackman, E. G. 1997, ApJ, 484, L79 
Chen, J. 2001, Space Sc. Rev., 95, 165

Choudhary, D. P., \& Gary, G. A. 1999, Sol. Phys., 188, 345

Choudhary, D. P., Sakurai, T., \& Venkatakrishnan, P. 2001, ApJ, 560, 439

Démoulin, P., Mandrini, C. H., Driel-Gesztelyi, L. V., et al. 2002 A\&A, 382, 650

Forbes, T. G., \& Isenberg, P. A. 1991, ApJ, 373, 294

Gibson, S. E., \& Low, B. C. 1998, ApJ, 493, 460

Gilbert, H, R., Holzer, T. E., Burkepile, J. T., \& Hundhausen, A. J. 2000, ApJ, 537, 503

Harvey, K. L. 1997, in Magnetic Reconnection in Solar Atmosphere, ed. R. D. Bentley, \& J. T. Mariska, ASP Conf. Ser., 111, 9

Hudson, H. S., Acton, L. W., \& Freeland, S. L. 1996, ApJ, 470, 629

Hudson, H. S. 1998, in Solar Physics with Radio Observations, Proc. Nobeyama Symp. 1998, NRO Rep. No. 479, ed. T. Bastian, N. Gopalswamy, \& K. Shibasaki, 159

Hudson, H. S., Labonte, B. J., Sterling, A. C., \& Watanabe, T. 1998, in Observational Plasma Astrophysics: Five Years of Yohkoh and Beyond, ed. T. Watanabe, T. Kosugi, \& A. C. Sterling (Kluwer Academic Publishers), 237
Kahler, S.W. 1992, ARA\&A, 30, 113

Khan, J. I., \& Hudson, H. S. 2000, GRL, 27, 1083

Lara, A., Gopalswamy, N., \& DeForest, C. 2000, GRL, 27, 1435

MacQueen, R. M., \& Fisher, R. R. 1983, Sol. Phys., 89, 89

Manchester, W. 2001, ApJ, 547, 503

Moore, R. L., Sterling, A. C., Hudson, H. S., \& Lemen, J. R. 2001, ApJ, 552, 833

Plunkett, S. P., Brueckner, G. E., Dere, K. P., et al. 1997, Sol. Phys., 175,699

Sheeley, N. Jr., Howard, R. A., Koomen, M. J., \& Michels, D. J. 1983, ApJ, 272, 349

Simnett, G. M. 1981 Proc. of 17th International Cosmic Ray Conf., Paris, France, July 13-25, 1981

Smith, Z., Watari, S., Dryer, M., et al. 1997 Sol. Phys., 171, 177

Sterling, A. C., \& Hudson, H. S. 1997 ApJ, 491, L55

Subramanian, P., \& Dere, K. P. 2001, ApJ, 561, 372

Svestka, Z. 2001, Space Sc. Rev., 95, 135

Tokman, M., \& Bellan, P. M. 2002, ApJ, 567, 1202 